\title{
A New Method to Separate Ground Clutter and Atmospheric Reflections in the Case of Similar Doppler Velocities
}

\author{
Dmitri Moisseev, Student Member, IEEE, Christine Unal, Herman Russchenberg, and \\ Leo Ligthart, Senior Member, IEEE
}

\begin{abstract}
This paper introduces a new ground clutter suppression technique which preserves weather echoes. This clutter suppression method uses both statistical and polarimetric properties of the target and clutter. This technique is intended for use in atmospheric studies for weather echoes the spectral properties of which do not differ much from those of ground clutter. This technique can be applied both to the total signal or to its separate Doppler frequency components.
\end{abstract}

Index Terms-Atmospheric radars, ground clutter suppression, polarimetry.

\section{INTRODUCTION}

$\mathbf{T}$ HE performance of a ground-based atmospheric radar is highly affected by ground clutter. Several clutter suppression techniques are used to reduce this influence. The most common ground-clutter suppression technique uses Doppler power spectrum information, that is, the mean Doppler velocity and the spectrum width [1]. Ground echoes have a zero mean Doppler velocity and a narrow Doppler spectrum and hence, reflections that have velocities close to zero are suppressed. It is more difficult to suppress the ground clutter if atmospheric targets have low radial velocities. Such targets, e.g., clouds, also have Doppler velocities close to zero and narrow Doppler spectra. Another approach to clutter suppression is to use the polarization diversity to identify range resolution cells which are contaminated by ground clutter. For this approach different polarimetric parameters can be used, such as: co-polar correlation coefficient [2], [3], the degree of polarization and circular depolarization ratio [4], linear depolarization ratio [5], and differential reflectivity [6]. These techniques are only used to detect echoes contaminated by ground clutter and not to separate weather echoes from clutter in a mixture. An attempt to improve current clutter suppression techniques using both Doppler and polarimetry was made by Moisseev et al. [7]; however, this technique also led to the loss of target information if target reflections and clutter occupied the same area in the Doppler spectrum.

This paper introduces a new clutter suppression technique, which improves the signal-to-clutter ratio even if ground

Manuscript received September 21, 2000; revised June 8, 2001.

The authors are with the International Research Centre for Telecommunications-Transmission and Radar, Delft University of Technology, 2628 CD Delft, The Netherlands (e-mail: D.Moisseev@its.tudelft.nl).

Publisher Item Identifier S 0196-2892(02)01884-3. clutter and weather echoes have similar Doppler velocities. The technique is optimal for nonscanning atmospheric radars. It improves the signal-to-clutter ratio with almost no effect on the reflections from weather targets.

Generally, waves scattered from radar objects are partially polarized. It is convenient to analyze partially polarized waves as a sum of two components: a stable and a fluctuating component. The stable component of the wave is scattered by targets that are constant over the observation time, e.g., buildings, the earth's surface, etc.; these contribute to the ground clutter. The fluctuating component of the scattered radar wave consists of reflections from weather objects and the fluctuating part of the ground clutter, such as trees and grass; also reflections from the stable targets can fluctuate if the propagation path is changing. Since the stable and the fluctuating parts of the wave contain reflections of different types of ground clutter, in this paper we will consider these two parts separately.

Section II discusses the first step of the processing: the suppression of stable clutter, which consists of removing a constant component of the reflected signals [1]. When atmospheric targets have Doppler velocities close to zero, a part of the reflections can appear to be reflections from nonfluctuating targets and thus be affected by the stable clutter suppression. This technique is equivalent to the suppression of the zero Doppler velocity cell. The width of the cell is defined by the averaging time over which the stable signal is calculated and thus the width can be manipulated to preserve weather signals. In this section, the influence of stationary-clutter suppression on atmospheric targets is discussed.

Section III introduces a new method for the suppression of the fluctuating part of ground clutter. This step of the processing is based on polarimetric differences between atmospheric targets and the fluctuating part of the ground clutter. To improve the use of this clutter suppression for meteorological studies, Doppler polarimetry is introduced in Section IV.

For this research, we used measurement data from the Delft Atmospheric Research Radar (DARR), the specifications of which are given in Table I. DARR is an frequency modulated continuous wave (FM-CW) S-band radar located on the roof of the $92 \mathrm{~m}$ high building of the Faculty of Information Technology and Systems. DARR is capable of carrying out Doppler and polarimetric target measurements simultaneously. Our clutter suppression method is illustrated with two precipitation measurements. In the first case, the precipitation event consists of three different groups of atmospheric targets, namely, a 
TABLE I

SPECIFICATIONS OF THE DELFT ATMOSPHERIC RESEARCH RADAR

\begin{tabular}{l|l}
\hline Radar parameter & Value \\
\hline \hline Radar frequency & $3.3 \mathrm{GHz}$ \\
\hline Antenna beamwidth & $1.8^{0}$ \\
\hline Frequency modulation law & triangular \\
\hline Number of samples per sweep & 128 \\
\hline Frequency deviation & $2 \mathrm{MHz}$ \\
\hline Sweep time & $1.25 \mathrm{~ms}$ \\
\hline Maximum range & $9600 \mathrm{~m}$ \\
\hline Range resolution & $75 \mathrm{~m}$ \\
\hline Polarization & linear \\
\hline Cross-polar isolation (for distributed targets) & $27 \mathrm{~dB}$ \\
\hline Receiver & one channel \\
\hline Measurement time of the scattering matrix & $3.75 \mathrm{~ms}$ \\
\hline Maximum unambiguous Doppler velocity & $6 \mathrm{~m} / \mathrm{s}$ \\
\hline Doppler velocity resolution & $4.7 \mathrm{~cm} / \mathrm{s}$ \\
\hline Observation time for one Doppler spectrum & $0.96 \mathrm{~s}$ \\
\hline Number of Doppler spectra used for averaging & 8 \\
\hline
\end{tabular}

precipitating cloud, a melting layer of the precipitation (bright band), and rain. Reflections from the melting layer show relatively strong polarimetric behavior in comparison with the other weather echoes. The second precipitation measurement is a drizzle measurement, in this case the atmospheric object of interest (drizzle) contains mainly spherical (polarimetrically isotropic) hydrometeors. For the drizzle measurement, the radar is pointed to the zenith; for the first measurement the radar had a $20^{\circ}$ elevation angle with the respect to the horizon. Thus, in both cases, the ground clutter reflections were mainly due to the antenna side lobes.

\section{SUPPRESSION OF STABLE CluTter}

Suppression of stable clutter is based on the fact that the correlation time of clutter is much longer than the correlation time of atmospheric targets. Therefore, if coherent averaging is performed over a time period $T_{o b s}$, which is much longer than the correlation time of atmospheric targets, the reflections from the atmosphere will be canceled and only reflections from the stable part of the ground clutter will remain. Subtraction of these averaged reflections from measurement data will cancel stable clutter. This processing is equivalent to the suppression of the zero Doppler velocity resolution cell, the size of the cell in this case is given by the averaging time as $\Delta v=\lambda /\left(2 T_{\text {obs }}\right)$.

During the Dutch national measurements campaign CLouds And RAdiation (CLARA) [8] extensive information about cloud physics was collected. It was measured that typical Doppler spectra widths for zenith radar measurements of clouds are between 0.18 and $0.3 \mathrm{~m} / \mathrm{s}$ [9]. It is known that the spectrum width of light rain caused by different fall velocities of hydrometeors is about $1 \mathrm{~m} / \mathrm{s}$ [1]. The Doppler spectrum width of a weather echo is a function of both the radar parameters, e.g., beam width, and of meteorological parameters that describe the time behavior of the ensemble of hydrometeors. The above-mentioned values are given for zenith radar measurements. Radar reflections measured at other elevation angles will have a different correlation time, since the part of the Doppler spectrum width that is due to

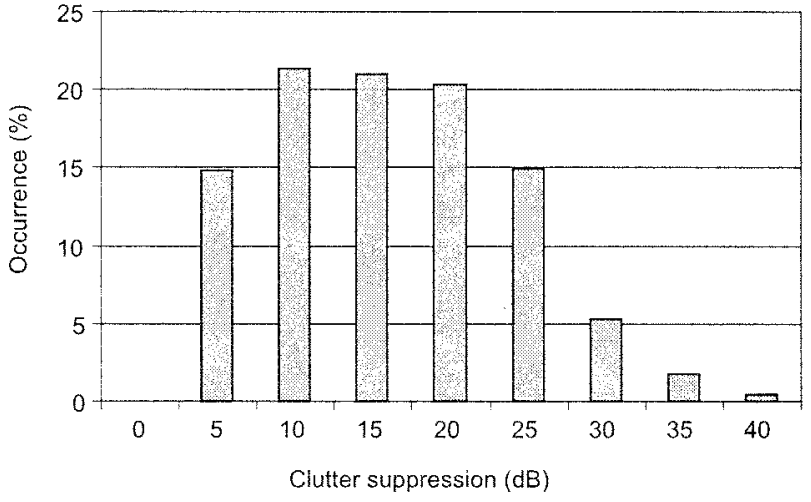

Fig. 1. Reduction of ground-clutter power due to the stable clutter suppression.

different fall velocities of hydrometeors depends on the elevation angle of the radar. However, these values give an estimate of a Doppler spectrum width for typical atmospheric targets.

If the information from atmospheric targets is to be preserved, the averaging time, which is used to calculate the stable clutter component, should be so long that the effective width of the Doppler cell is much smaller than the Doppler width of weather echoes (in our case, $0.18 \mathrm{~m} / \mathrm{s}$ ).

Fig. 1 gives the result of the stable clutter suppression. Ground-clutter reflections before and after clutter suppression are calculated and a histogram of the power ratios is given. These ground-clutter measurements were performed by DARR for different radar elevation angles and in different azimuth directions. The wind speed for all these measurements was between 3.5 and $8 \mathrm{~m} / \mathrm{s}$. In all analyzed case, ground clutter can be considered as an urban-area ground clutter. About $8 \mathrm{~s}$ of data were used to calculate the average signal for processing. This averaging time gives Doppler cells effective width of $5.9 \cdot 10^{-3} \mathrm{~m} / \mathrm{s}$, since $\lambda=0.09 \mathrm{~m}$ and the observation time $T_{s}$ is $7.96 \mathrm{~s}$. Thus, the influence of this processing on the atmospheric targets signal is negligible.

The median value for the stable clutter suppression is about $13 \mathrm{~dB}$. The spread in the histogram is caused by the presence of data with different ground clutter content. High values of clutter suppression correspond to ground clutter with a large stable part, which corresponds to the reflections from man made objects. Low values of the clutter suppression, on the other hand, correspond to clutter with a relatively large fluctuating part, which corresponds to natural objects such as trees.

\section{Fluctuating Ground Clutter SuPPRESSION}

\section{A. Short Review of the Theory of Polarimetry}

The electric field vector of a wave scattered from a target is related to the transmitted wave electric field vector by the scattering matrix of the target as

$$
\left(\begin{array}{c}
E_{x} \\
E_{y}
\end{array}\right)=\left(\begin{array}{ll}
S_{x x} & S_{x y} \\
S_{y x} & S_{y y}
\end{array}\right)\left(\begin{array}{c}
E_{x}^{t} \\
E_{y}^{t}
\end{array}\right)
$$

where $(x, y)$ is an arbitrary orthonormal polarization basis. In most cases, a radar target fluctuates over time, which implies 
changes of the scattering matrix and of the scattered wave. To describe a time-dependent wave, the coherence matrix is used

$$
\begin{aligned}
& \mathbf{J}(\tau)=\left\langle( \begin{array} { l } 
{ E _ { x } ( t ) } \\
{ E _ { y } ( t ) }
\end{array} ) \left(\begin{array}{ll}
E_{x}^{*}(t+\tau) & \left.\left.E_{y}^{*}(t+\tau)\right)\right\rangle
\end{array}\right.\right. \\
& =\left(\begin{array}{cc}
\left\langle E_{x}(t) \cdot E_{x}^{*}(t+\tau)\right\rangle & \left\langle E_{x}(t) \cdot E_{y}^{*}(t+\tau)\right\rangle \\
\left\langle E_{y}(t) \cdot E_{x}^{*}(t+\tau)\right\rangle & \left\langle E_{y}(t) \cdot E_{y}^{*}(t+\tau)\right\rangle
\end{array}\right)
\end{aligned}
$$

where \langle\rangle denotes ensemble averaging and $\tau$ is the time delay. The wave behavior is assumed to be a stationary random process. For the case of quasi-monochromatic waves: if the time delays $\tau$ are restricted by $|\tau| \ll 1 / \sigma$, where $\sigma$ is the width of the spectrum of the scattered wave, e(2) will become [10]

$$
\begin{aligned}
\mathbf{J}(\mathbf{0}) & =\left(\begin{array}{ll}
\left\langle E_{x}(t) E_{x}^{*}(t)\right\rangle & \left\langle E_{x}(t) E_{y}^{*}(t)\right\rangle \\
\left\langle E_{y}(t) E_{x}^{*}(t)\right\rangle & \left\langle E_{y}(t) E_{y}^{*}(t)\right\rangle
\end{array}\right) \\
& =\left(\begin{array}{ll}
J_{x x} & J_{x y} \\
J_{y x} & J_{y y}
\end{array}\right) .
\end{aligned}
$$

This expression gives a more common notation of the wave coherence matrix and is widely used in radar polarimetry [11].

For a completely unpolarized wave, the coherence matrix $\mathbf{J}$ will become a diagonal matrix, since an unpolarized wave has no preferable polarization, which implies that $\left\langle\left|E_{x}\right|^{2}\right\rangle=\left\langle\left|E_{y}\right|^{2}\right\rangle$ and $\left\langle E_{x} E_{y}^{*}\right\rangle=0$. A partially polarized wave can be represented as a sum of a completely polarized and completely unpolarized waves; in mathematical form it can be written as [12]

$$
\mathbf{J}=\left(\begin{array}{cc}
A & 0 \\
0 & A
\end{array}\right)+\left(\begin{array}{cc}
B & J_{x y} \\
J_{y x} & C
\end{array}\right)
$$

where the first matrix represents the coherence matrix of the completely unpolarized wave and the second one that of the completely polarized wave. Following a well-known procedure [10], [12] using the diagonalization of $\mathbf{J}$ we can find that

$$
\begin{aligned}
& A=\frac{1}{2}\left(\operatorname{Tr} \mathbf{J}-\sqrt{T r^{2} \mathbf{J}-4 \operatorname{det} \mathbf{J}}\right) \\
& B=\frac{1}{2}\left(J_{x x}-J_{y y}\right)+\frac{1}{2} \sqrt{T r^{2} \mathbf{J}-4 \operatorname{det} \mathbf{J}} \\
& C=\frac{1}{2}\left(J_{y y}-J_{x x}\right)+\frac{1}{2} \sqrt{T r^{2} \mathbf{J}-4 \operatorname{det} \mathbf{J}}
\end{aligned}
$$

where $\operatorname{Tr} \mathbf{J}$ denotes the trace of the matrix $\mathbf{J}$ and $\operatorname{det} \mathbf{J}$ denotes determinant of $\mathbf{J}$. The degree of polarization $p$ of a wave is the ratio of the power in the polarized component of the wave $I_{\mathrm{pol}}$ and the total power of the wave $I_{\text {tot }}$. It can be written as

$$
\begin{aligned}
p & =\sqrt{1-\frac{4 \operatorname{det} \mathbf{J}}{T^{2} \mathbf{J}}} \\
& =\sqrt{\frac{\left(J_{x x}-J_{y y}\right)^{2}+4 \cdot J_{x x} J_{y y}}{J_{x x}+J_{y y}}} \\
& =\frac{I_{\text {pol }}}{I_{\text {tot. }}}
\end{aligned}
$$

For an unpolarized wave $p=0$, since $(4 \operatorname{det} \mathbf{J}) /\left(\operatorname{Tr}^{2} \mathbf{J}\right)=1$ or $J_{x y}=J_{y x}=0$ and $J_{x x}=J_{y y}$. For a completely polarized wave $p=1$, since $\operatorname{det} \mathbf{J}=0$.

\section{B. Atmospheric Targets and Fluctuating Ground Clutter}

Most of the atmospheric targets are characterized by the fact that cross-polar reflections in the $h-v$ linear polarization basis are much smaller than co-polar ones. For light rain and water clouds $L d r=\left\langle\left|S_{h v}\right|^{2}\right\rangle /\left\langle\left|S_{h h}\right|^{2}\right\rangle$ is usually smaller than $-30 \mathrm{~dB}$, but for the melting layer of a precipitation event, this value can lie in the order of $-15 \mathrm{~dB}$ [5]. It should be mentioned that the $L d r$ values in a melting layer belong to the highest values to be found in weather echoes. It is common to assume that the co-polarized and cross-polarized elements of the scattering matrix for ground clutter are uncorrelated [13], [14]. In the case of precipitation measurements, it was recently shown [15] that the cross correlation coefficient between these scattering matrix elements can be close to unity. However, in this paper, the assumption that the co-polarized and cross-polarized elements of the scattering matrix of precipitation are uncorrelated is used since it represents the less favorable case for the proposed clutter suppression method. Then, according to (1) and (3), the coherence matrix of an atmospheric target, expressed in $h-v$ linear polarization basis, when the transmitted wave is horizontally polarized, will be

$$
\mathbf{J}=\left(\begin{array}{cc}
\left\langle\left|S_{h h}\right|^{2}\right\rangle & 0 \\
0 & \left\langle\left|S_{h v}\right|^{2}\right\rangle
\end{array}\right)
$$

and the degree of polarization according to (6) will be

$$
p=\left|\frac{(1-L d r)}{(1+L d r)}\right|
$$

Strictly speaking, the matrix (7) is only proportional to the actual wave coherency matrix (3). However, we are only interested in relative polarimetric parameters and hence the coefficient of proportionality can be omitted here. Thus, for atmospheric targets, the limits of the degree of polarization will change between 0.94 , for a precipitation melting layer, and 1 for light rain and water clouds.

As was shown in the beginning of this paper, stable clutter suppression removes the monochromatic component of the wave scattered from the ground clutter. This component dominates reflections from the ground clutter and its suppression gives a reduction by $13 \mathrm{~dB}$ of the clutter signal. The residue part of the clutter varies over time and a wave reflected from it has nonzero spectrum width. These reflections come from small objects which fluctuate over time, i.e., trees, grass, etc. A theoretical prediction of the degree of polarization for such targets is complicated. It is even more complicated by the fact that in most of the cases reflections from the ground clutter are obtained via the antenna side lobes. Thus, the exact polarization basis of the radar sounding is unknown and antenna patterns for different polarizations are different.

Measurements of the degree of polarization of atmospheric targets as rain, precipitating cloud and melting layer of precipitation as well as data of the ground clutter were obtained during a measurements campaign in November 1997. These measurements were carried out using DARR. Scattering matrices of the precipitation were obtained in $h-v$ linear polarization basis. 


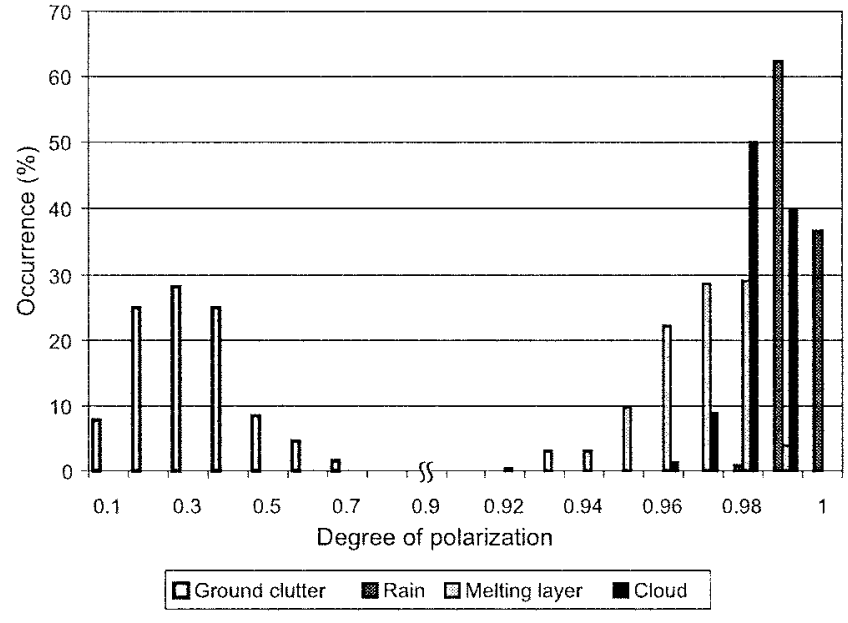

Fig. 2. Degree of polarization for ground clutter, rain, melting layer, and cloud.

Since the exact polarization states of the side lobes of the antenna are unknown, reflections from the ground clutter were treated as if they were also obtained in $h-v$ linear polarization basis. It should be noted that even though the degree of polarization can change from basis to basis, it mainly depends on the time variations of the backscattered wave, which are defined by the variations of the target. The radar elevation angle for these measurements was $20^{\circ}$ with respect to the horizon. To calculate the degree of polarization, approximately $8 \mathrm{~s}$ of data were used. Prior to the calculation of the degree of polarization the stable clutter was suppressed. This processing removes the constant component of the signal, which is completely polarized by definition.

Measurements results are presented in Fig. 2. As can be seen the degree of polarization for the atmospheric targets is close to unity, as expected. The mean value for the degree of polarization of clouds and rain is higher than 0.98 . This value is expected to be an underestimation since cross-polar measurements of rain and water clouds are affected by the coupling of the polarization channels [16]. The cross-polar isolation of DARR for distributed targets is $-27 \mathrm{~dB}$. The mean value of the degree of polarization of the melting layer is 0.97 . This value is defined by physical processes in the melting layer itself, since the cross-polar reflections are about $10 \mathrm{~dB}$ higher than the antenna limit.

Fig. 2 also shows that the mean value of the degree of polarization of the ground clutter is about 0.3. That means that the waves scattered from the ground clutter are mainly depolarized. However, values of the degree of polarization for ground clutter were reported to be close to unity in [17]. These measurements were performed in the suburbs of Rome, Italy. Since in both cases we deal with the same type of ground clutter (urban area ground clutter), differences in their physical properties cannot explain the discrepancy between the results. However, the difference between these and our results can be explained by the fact that reflections from an urban area are dominated by the reflections from the stable ground clutter. Furthermore, in our case, prior to the calculation of the degree of polarization, the stable ground clutter was suppressed and hence the completely polarized part of the ground clutter was removed. This part dominates the ground clutter reflections; it is $13 \mathrm{~dB}$ larger than the fluctuating part of ground clutter. Thus, this difference in the processing can explain the difference in results.

The degree of polarization gives a high contrast between ground clutter and weather echoes, as can be seen in Fig. 2 . That gives us the possibility to use the degree of polarization for the detection of the range resolution cells which are affected by ground clutter. This approach was already discussed by Da Silveira and Holt [4]. In this paper, the difference in polarimetric properties of weather echoes and fluctuating ground clutter for clutter suppression is used by applying the wave decomposition theorem. The degree of polarization for ground clutter has the median value of 0.3 and for atmospheric targets the value of 0.97. Now, if the backscattered wave is decomposed in completely polarized and completely unpolarized waves, the amount of clutter power which will remain in the polarized part will be on average $5.2 \mathrm{~dB}$ lower than that in the backscattered wave. Moreover, the power in the completely polarized part of the atmospheric signal will only be reduced by $0.13 \mathrm{~dB}$.

The above given values of the polarimetric clutter suppression correspond to the reduction of the clutter power in the backscattered wave. The gain in the signal-to-clutter ratio for the co-polar element of the scattering matrix can be obtained from (8) assuming that $J_{x y}=J_{y x}=0$. In this case, the reduction of clutter is given by (1-Ldr), where $L d r$ is obtained from (8). This leads us to the final values of the fluctuating clutter suppression using the wave decomposition theorem, which on average give 3.35 $\mathrm{dB}$ of ground clutter suppression and will reduce power in atmospheric signal by only $0.07 \mathrm{~dB}$.

\section{DOPPLER POlarimetry AND WaVe Decomposition}

The combination of the stable clutter suppression and wave decomposition is an attractive tool for ground clutter suppression, as it results in about $16 \mathrm{~dB}$ of clutter suppression and preserves reflections from the atmospheric targets. The drawback of this method is that the output of this processing gives averaged intensities, so the Doppler spectrum information is lost. The solution to this problem lies in applying the method to Doppler spectra.

Let us introduce the spectral coherence matrix $\mathbf{F}(\omega)$ [10], which is analogous to the wave coherence matrix (3) but defined for each Doppler frequency

$$
\mathbf{F}(\omega)=\left(\begin{array}{ll}
F_{x x}(\omega) & F_{x y}(\omega) \\
F_{y x}(\omega) & F_{y y}(\omega)
\end{array}\right)
$$

where

$$
F_{i j}(\omega)=2 \int_{-\infty}^{+\infty} J_{i j}(\tau) \exp (-i \omega \tau) d \tau
$$

The expression (10) relates the wave coherence matrix (2) to the spectral coherence matrix (9).

The relation between the matrix $\mathbf{J}(0)$ and the spectral coherence matrix is

$$
J_{i j}(0)=\frac{1}{4 \pi} \int_{-\infty}^{+\infty} F_{i j}(\omega) d \omega
$$




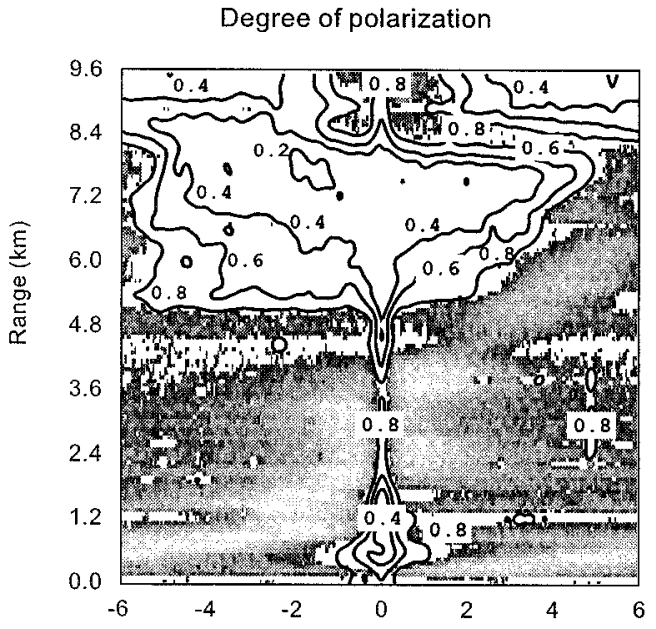

a).

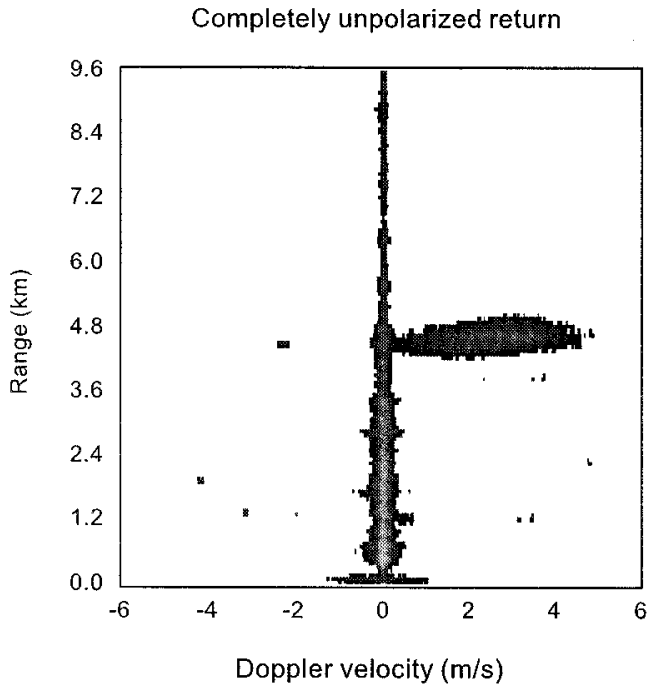

c).

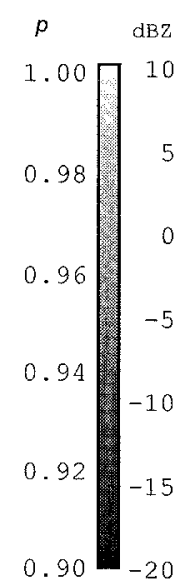

$\mathrm{dBZ}$

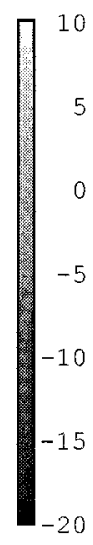

HH-polarization before decomposition

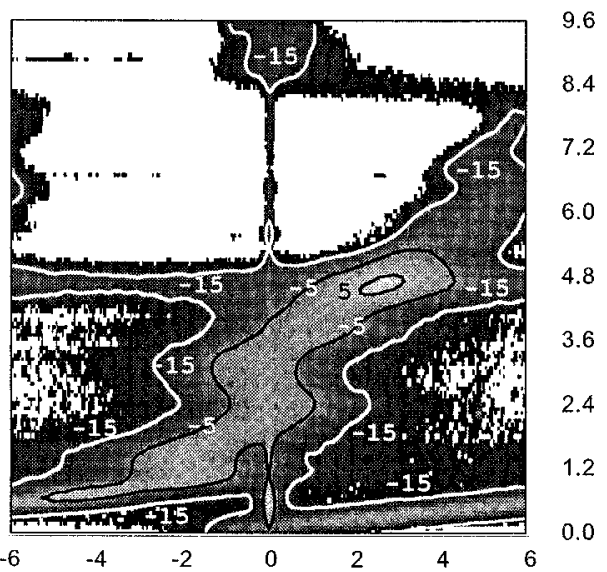

b).

Completely polarized return - $\mathrm{HH}$

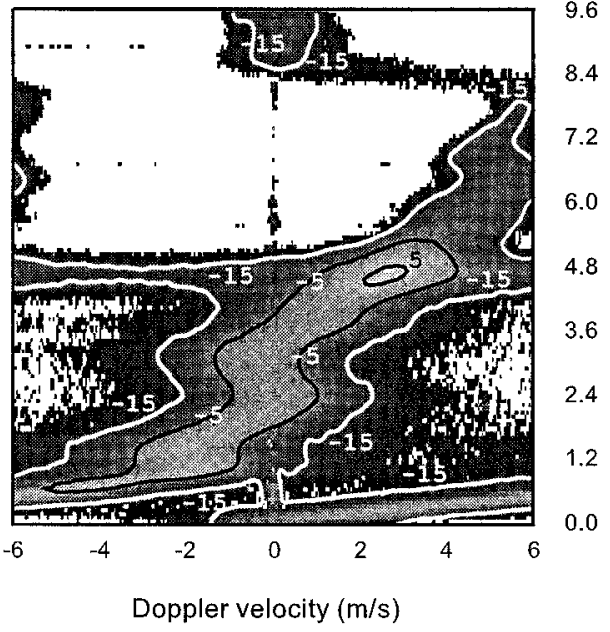

d).

Fig. 3. Slant profile of precipitation, $20^{\circ}$ elevation.

In the discrete form, if we take into account that the measurement time $T$ is finite, (11) can be rewritten as [18]

$$
J_{i j}(0)=\frac{1}{4 \pi} \sum_{k} F_{i j}^{(T)}\left(\omega_{k}\right) \cdot \Delta \omega
$$

where $\Delta \omega \sim 1 / T$ denotes the spectral (Doppler) frequency resolution. Thus a wave coherence matrix is just the sum of coherence matrices of waves with frequencies $\omega_{k}$ and the spectrum width $\Delta \omega$. This result is logical since waves with different center frequencies are statistically independent and their cross correlations are zero.

To clarify the physical meaning of (12), we consider two limiting cases.

1) If the observation time is small $\left(T \ll 1 / \sigma_{d}, \sigma_{d}\right.$ is the width of the Doppler spectrum), then the Doppler frequency resolution cell contains the complete Doppler spectrum of targets. Therefore, the wave coherence matrix $\mathbf{J}(0)$ will coincide with the spectral coherence matrix $\mathbf{F}(\omega)$. In this case, application of the wave de- composition theorem to the spectral coherence matrix will give the same results as its application to the wave coherence matrix.

2) An infinitely long observation time will lead to infinitesimal Doppler frequency resolution cells and hence every Doppler cell will represent a monochromatic wave. For example, if a wave consists of two equal intensity components with slightly different frequencies and having orthogonal polarizations, then according to the Stokes theorem [9] this wave will behave as a completely unpolarized wave. However, if spectral coherence matrices are calculated for a time interval that is long enough to separate these components of the wave, then the resulting two spectral coherence matrices will represent completely polarized waves. In this case, application of the wave decomposition theorem in the time domain and in the frequency domain will give two completely different results.

Thus, to be able to apply the wave decomposition theorem to the Doppler spectrum, we should have Doppler resolution 


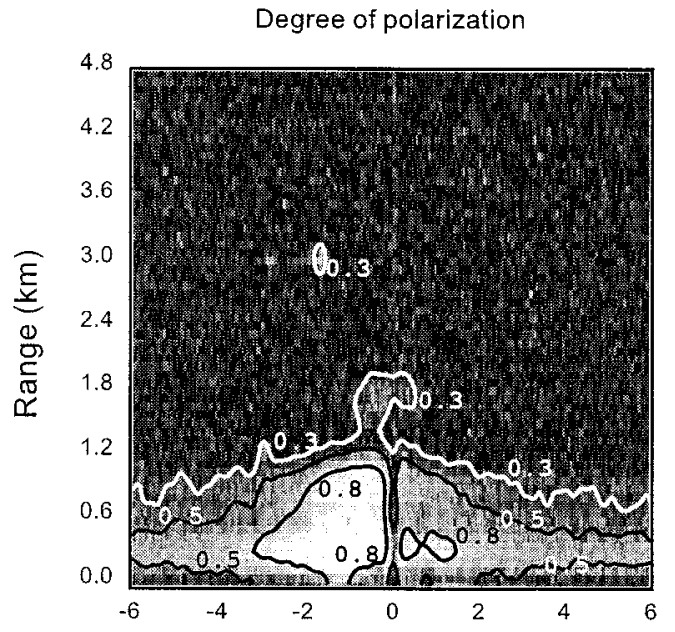

a).

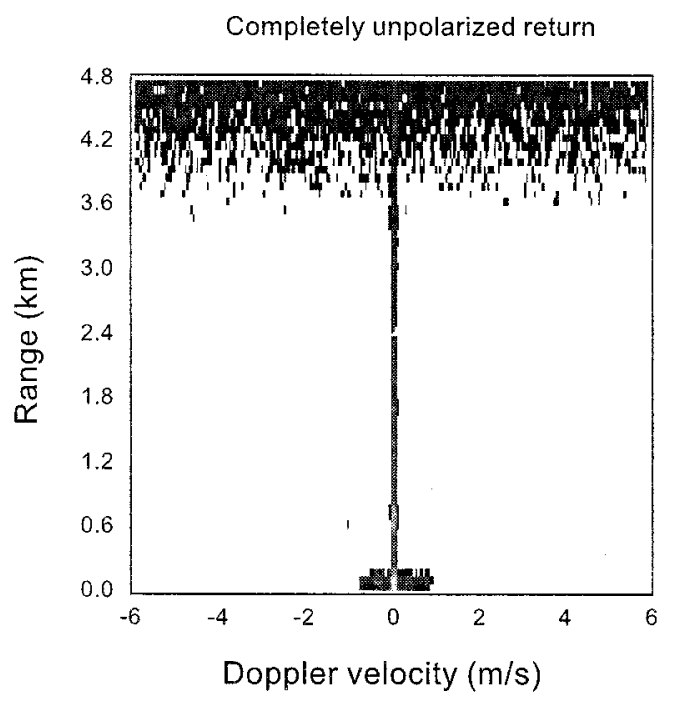

c).
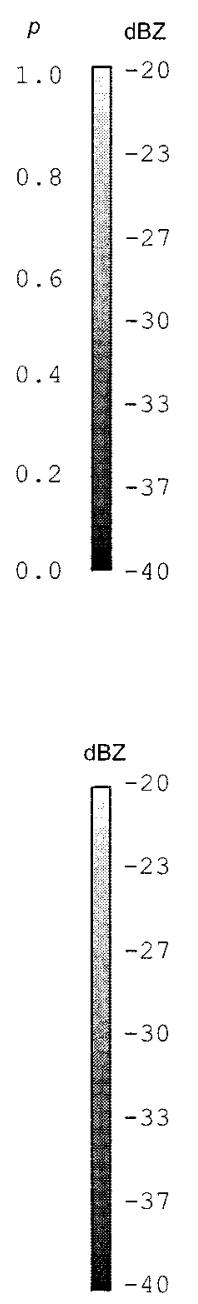

Fig. 4. Vertical profile of precipitation (drizzle).
HH-polarization before decomposition

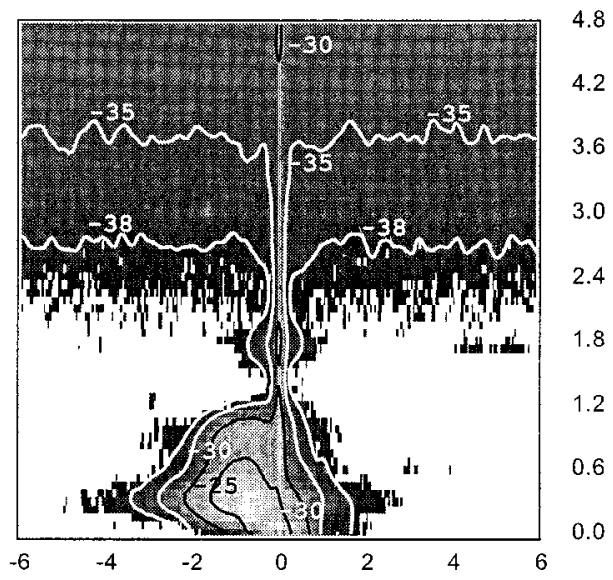

b).

Completely polarized return - $\mathrm{HH}$

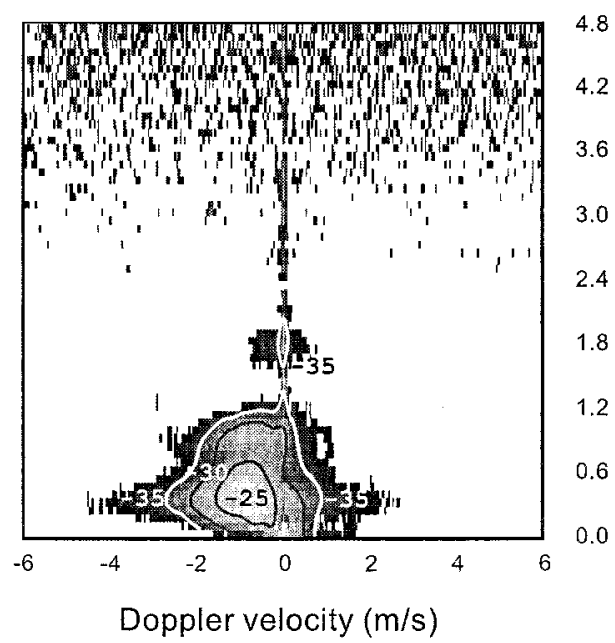

d). cells that are large enough to contain the complete spectrum of the ground clutter. The Doppler spectrum resolution should be high enough to allow for a good estimation of widths and mean velocities of atmospheric targets.

\section{Results of the SPectral Coherence MATRIX DECOMPOSITION}

To test this new clutter suppression technique, we have measured slant and vertical profiles of precipitation. The elevation angle of the radar for the slant profile measurement was $20^{\circ}$ relative to horizon. The co-polar and cross-polar components of the scattered wave were measured by alternating linear polarizations on the receiving channel; the polarization state is switched every $1.25 \mathrm{~ms}$. For both examples, $1 \mathrm{~min}$ of data is collected. Prior to the Doppler processing, stable clutter suppression is performed. Coherent averages of the co-polar and cross-polar echoes for the stable clutter suppression are computed using the complete data set. It is important to note that the coherence time of DARR is in the order of $3 \mathrm{~min}$. The radar coherence time is inversely proportional to the spectrum width caused by the radar phase instability.

The Doppler spectrum of the coherence matrix is obtained each $0.96 \mathrm{~s}$ using 256 samples. The maximum unambiguous Doppler velocity for this setting is $6 \mathrm{~m} / \mathrm{s}$ and the Doppler velocity resolution is $0.047 \mathrm{~m} / \mathrm{s}$. This number of samples is a trade off between a high Doppler resolution for the retrieval of spectrum information on weather echoes and relatively large resolution cells to contain most of the ground clutter reflections in one Doppler cell. More details about Doppler polarimetric processing can be found in [20]. A total of eight spectra are obtained for each element of the coherence matrix. The number of spectra is a compromise between the good approximation of the degree of polarization and a good time resolution for real-time applications. Using (4), (5), (6), and (12), the degree of polarization, and the completely polarized and completely unpolarized returns are calculated for every Doppler resolution cell.

The slant and the vertical profile of precipitation are shown in Figs. 3 and 4, respectively. Fig. 3(b) and Fig. 4(b) show the 
reflectivity of the precipitation versus range and Doppler velocity. The high reflectivity, between -5 and $5 \mathrm{dBZ}$, at $4.8 \mathrm{~km}$ in Fig. 3(b), corresponds to the melting layer of the precipitation. Reflections below the melting layer come from rain. Ice crystals falling from the cloud cause reflections above the melting layer. Other features which can be clearly seen in Fig. 3(b) are measurement and processing artifacts. Such artifacts are spectral leakage and aliasing. The spectral leakage, caused by high reflection from the melting layer, can be clearly seen at a height of $4.8 \mathrm{~km}$; it occupies all Doppler velocities and has a lower reflectivity than the melting layer itself. Since the maximum unambiguous velocity for these measurements was $6 \mathrm{~m} / \mathrm{s}$, all velocities which exceed this limit are aliased. This effect can be seen in Fig. 3(b) at ranges between 0 and $1.2 \mathrm{~km}$ and Doppler velocities between -2 and $6 \mathrm{~m} / \mathrm{s}$. Fig. 4(b) shows the Doppler spectrum of drizzle. The low reflectivity signal, about $-38 \mathrm{dBZ}$, at $1.8 \mathrm{~km}$ comes from a cloud and reflections between 0 and 1.2 $\mathrm{km}$ are caused by the drizzle itself.

Fig. 3(a) and Fig. 4(a) show the degree of polarization versus range and Doppler velocity. In Fig. 3(a), the grayscale gives values of the degree of polarization between 0.9 and 1 and contours show values between 0 and 0.8 . As expected, reflections from the rain, the melting layer, and the cloud have a high degree of polarization values, which vary between 0.9 and 1 . Reflections from the ground clutter and noise have lower values of degree of polarization. It is interesting to compare the degree of polarization values for areas in Fig. 3(a) and Fig. 4(a) which have no significant reflections. In Fig. 3(a), the degree of polarization values for such an area (range interval 5-8 km and Doppler velocities interval from -5 until $-1 \mathrm{~m} / \mathrm{s}$ ) vary between 0.2 and 0.6 . These rather high values can be explained by the influence of spectral leakage. Therefore, these values correspond not only to noise but to a sum of noise and spectral leakage. As it can be seen in Fig. 4(a), areas which contain only noise have values for the degree of polarization between 0 and 0.2 .

Fig. 3(d) andFig. 4(d) show the completely polarized backscattering of the considered events. As can be seen, clutter reflections have been reduced by approximately $5 \mathrm{~dB}$, while reflections from atmospheric targets are hardly affected at all (a reduction of $0.1 \mathrm{~dB}$ can be expected in the melting layer). Completely unpolarized returns in Fig. 3(c) and Fig. 4(c) show the difference in reflection before and after fluctuating clutter suppression. Fig. 3(c) clearly shows that the main component of unpolarized reflections is clutter. However, it also shows that the lower part of the melting layer also contributes to unpolarized reflections. Thus, the melting layer reflections are affected by this clutter suppression, but this influence is not large and coincides with our estimation made above. Fig. 4(c) shows approximately the same picture, only more clearly. Note that due to the processing, noise is also suppressed.

\section{CONCLUSIONS}

It was shown that it is very useful to consider ground clutter as a sum of the stable and fluctuating (time-dependent) components. Moreover, the change of the polarimetric properties of ground clutter due to suppression of the stable part of the signal were also discussed. It was shown that the fluctuating part of ground clutter has low degree of polarization, while the degree of polarization of the complete clutter is close to unity. It was also shown that weather echoes have a high degree of polarization. Thus, the degree of polarization is a parameter that can be used to discriminate between atmospheric target and fluctuating ground clutter.

This study has led to a new ground clutter suppression technique that preserves reflections from atmospheric targets. This clutter suppression technique shows good performance. It allows for separation of ground clutter and atmospheric objects even if they occupy the same Doppler spectrum area. This processing gives $16.4 \mathrm{~dB}$ of clutter suppression on average.

This new clutter suppression method is mainly applicable to cloud radars and wind profilers, since it requires suppression of the stable clutter component, which is time-dependent in scanning weather radars. Nevertheless, the polarimetric processing which is discussed in this paper could be used to remove residuals of ground clutter, which are left after the standard Doppler processing in weather radars.

\section{ACKNOWLEDGMENT}

The authors would like to thank the anonymous reviewers for their constructive criticisms.

\section{REFERENCES}

[1] R. J. Doviak and D. S. Zrnic, Doppler Radar and Weather Observations. New York: Academic, 1993.

[2] A. V. Ryzhkov and D. S. Zrnic, "Polarimetric rainfal estimation in the presence of anomalous propagation," J. Atmos. Ocean. Technol., vol. 15, pp. 1320-1330, Dec. 1998.

[3] D. S. Zrnic and A. V. Ryzhkov, "Polarimetry for weather surveillance radars," Bull. Amer. Meteorol. Soc., vol. 80, pp. 389-406, Mar. 1999.

[4] R. B. Da Silveira and A. R. Holt, "A neural network application to discriminate between clutter and precipitation using polarization information as feature space," in Proc. 28th Conf. Radar Meteorology, Austin, TX, Sept. 7-12, 1997, pp. 57-58.

[5] M. Hagen, "Identification of ground clutter by polarimetric radar," in Proc. 28th Int. Conf. Radar Meteorology, Amer. Meteor. Soc., Austin, TX, Sept. 1997, pp. 67-68.

[6] J. Vivekanandan, D. S. Zrnic, S. M. Ellis, R. Oye, A. V. Ryzhkov, and J. Straka, "Cloud microphysics retrieval using S-band dual-polarization radar measurements," Bull. Amer. Meteorol. Soc., vol. 80, pp. 381-388, Mar. 1999.

[7] D. Moisseev, C. Unal, H. Russchenberg, and L. Ligthart, "Doppler polarimetric ground clutter identification and suppression for atmospheric radars based on co-polar correlation," in Proc. MIKON, Wroclaw, Poland, May 22-24, 2000, pp. 94-97.

[8] A. V. Lammeren, A. Feijt, D. Donovan, H. Bloemink, H. Russchenberg, V. Venema, J. Erkelens, A. Apituley, H. T. Brink, A. Khlystov, S. Jongen, G. Brussaard, and M. Herben, "CLARA: Clouds and radiation- intensive measurement campaigns in the netherlands," in Symp. Proc.: Remote Sensing of Cloud Parameters: Retrieval and Validation, Delft, The Netherlands, Oct. 1999, pp. 5-10.

[9] V. Venema, H. Russchenberg, and L. Ligthart, "Correction for clipping of doppler spectra from clouds and other atmospheric targets," in Proc. IGARSS'99 Symp., Hamburg, Germany, June-July 28-2, 1999, pp. 1180-1182.

[10] J. Perina, Coherence of Light. New York: Van Nostrand, 1972.

[11] F.T. Ulaby and C. Elachi, Radar Polarimetry for Geoscience Applications. Norwood, MA: Artech House, 1990.

[12] V. A. Potechin and V. N. Tatarinov, Theory of Electromagnetic Field Coherence (in Russian). Moscow: Izdatel'stvo Svjaz', 1978.

[13] M. Borgeaud, R. Shin, and J. Kong, "Theoretical models for polarimetric radar clutter," J. Electromagn. Waves Appl., vol. 1, pp. 67-86, 1987.

[14] A. A. Swartz, H. A. Yueh, J. A. Kong, L. M. Novak, and R. T. Shin, "Optimal polarizations for achieving maximum contrast in radar images," $J$. Geophys. Res., vol. 93, pp. 15 252-15 260, Dec. 1988. 
[15] A. V. Ryzhkov, "Interpretation of polarimetric radar covariance matrix for meteorological scatterers: Theoretical analysis," J. Atmos. Ocean. Technol., vol. 18, pp. 315-328, Mar. 2001.

[16] D. N. Moisseev, C. M. H. Unal, H. W. J. Russchenberg, and L. P. Ligthart, "Polarimetric radar calibration using distributed target," in Proc. IGARSS'99 Symp., Hamburg, Germany, June-July 28-2, 1999, pp. 434-436.

[17] D. Giuli, M. Fossi, and M. Gherardelli, "Polarization behavior of ground clutter during dwell time," Proc. Inst. Elect. Eng., vol. 138, pt. F, no. 3, pp. 211-217, 1991.

[18] W. B. Davenport and W. L. Root, An Introduction to the Theory of Random Signals and Noise. New York: McGraw-Hill, 1958.

[19] S. Chandrasekhar, Radiative Transfer. Oxford, U.K.: Oxford Clarendon, 1950

[20] C. M. H. Unal, D. N. Moisseev, and L. P. Ligthart, "Doppler-polarimetric measurements of precipitation," in Proc. JIPR, Nantes, France, July 1998, pp. 429-438.

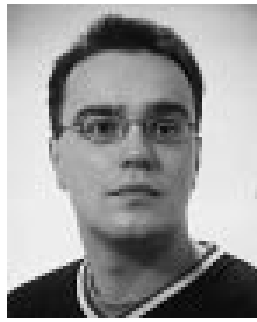

Dmitri Moisseev (S'98) received the M.S. degree from the Moscow Institute of Physics and Technology, Moscow, Russia, in 1997. He is currently pursuing the Ph.D. degree at the Delft University of Technology, Delft, The Netherlands, in the field of radar polarimetry. His research interests include radar polarimetry and SAR interferometry.

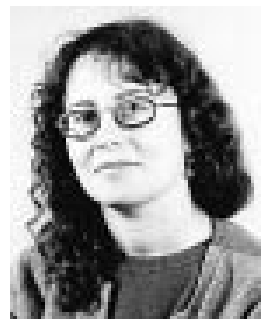

Christine Unal received the D.E.A. degree in physics for remote sensing from the University of Paris, Paris, France, in 1987.

She joined the Delft University of Technology, Delft, The Netherlands, in 1988, where she is a Research Scientist. She has been working on microwave remote sensing and her current research field is in radar polarimetry.

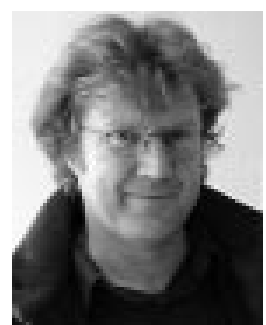

Herman Russchenberg has been involved in radar remote sensing of the atmosphere since 1986. At that time, the emphasis was put on the study of precipitation and the melting layer in stratiform rain clouds. Over the years, the emphasis has moved toward the remote sensing of clouds and clear air. He is experienced in theoretical as well experimental research of the scattering process and the retrieval of geophysical parameters from radar measurements. He is currently with the International Research Centre for Telecommunications-Transmission and Radar, Delft University of Technology, Delft, The Netherlands, where he heads the remote sensing sector.

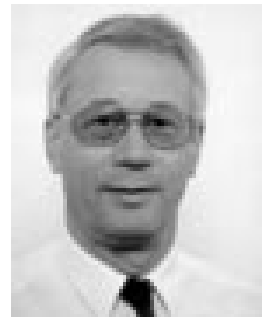

Leo Ligthart (M'94-SM'95) was born in Rotterdam, The Netherlands, on September 15, 1946. $\mathrm{He}$ received the engineer's degree (cum laude) and a Dr.Tech degree from Delft University of Technology, Delft, The Netherlands in 1969 and 1985, respectively, and the Ph.D. degrees (honoris causa) from Moscow State Technical University of Civil Aviation, Moscow, Russia, in 1999, and Tomsk State University of Control Systems and Radioelectronics, Tomsk, Russia, in 2001.

Since 1992, he has held the Chair of Microwave Transmission, Radar, and Remote Sensing in the Department of Information Technology and Systems, Delft University of Technology, Delft, The Netherlands. In 1994, he became Director of the International Research Center for Telecommunications-Transmission and Radar. His principal areas of specialization include antennas and propagation and radar and remote sensing, but he has also been active in satellite, mobile, and radio communications. 